\title{
CONCERNING THE DEFINITION OF HARMONIC FUNCTIONS
}

\author{
E. F, BECKENBACH
}

1. Introduction. A real function $u(x, y)$, defined in a domain (nonnull connected open set) $D$, is said to be harmonic in $D$ provided $u(x, y)$ and its partial derivatives of the first and second orders are continuous and the Laplace equation,

$$
\Delta u \equiv \partial^{2} u / \partial x^{2}+\partial^{2} u / \partial y^{2}=0,
$$

is satisfied throughout $D$. A function is said to be harmonic at a point provided it is harmonic in a domain containing the point.

It has been shown [1 $]^{1}$ that if $u(x, y)$ is continuous in $D$ and if the second order partial derivatives $\partial^{2} u / \partial x^{2}$ and $\partial^{2} u / \partial y^{2}$ exist and satisfy the Laplace equation (1) throughout $D$, then $u(x, y)$ is harmonic in $D$.

We shall show that if $u(x, y)$ and its partial derivatives $\partial u / \partial x$ and $\partial u / \partial y$ are continuous in $D$, if $\partial u / \partial x$ and $\partial u / \partial y$ are differentiable, or even have finite Dini derivates, with respect to $x$ and $y$ at all points of $D$ except at most at the points of a denumerable set of points in $D$, and if the Laplace equation (1) is satisfied at almost all points of $D$ at which $\partial^{2} u / \partial x^{2}$ and $\partial^{2} u / \partial y^{2}$ exist, then $u(x, y)$ is harmonic in $D$.

Our result is comparable with the Looman-Menchoff theorem [3, pp. 9-16; 5, pp. 198-201] concerning the Cauchy-Riemann first order partial differential equations and analytic functions of a complex variable. Ridder [4] has stated that harmonic functions can be given a Looman-Menchoff characterization; but a generalization of the Looman-Menchoff theorem on which his proof is based is invalid, for there are functions having isolated singularities which satisfy the hypotheses of the generalization without satisfying the conclusion. For a generalization of the Looman-Menchoff theorem, see Maker [2].

2. Notation and lemmas. By $C(Q)$ we shall denote a square, by $C(R)$ a rectangle, having sides parallel to the coordinate axes. The set consisting of the points of $C(Q)$, or of $C(R)$, plus its interior, will be denoted by $Q$, or $R$, respectively.

Let $F$ be a non-null set closed with respect to the domain $D$, and $C(Q)$ any square with $Q$ lying in $D$, with sides of positive length and parallel to the coordinate axes, and with center at a point of $F$. Then the points common to $F$ and $Q$ will be called a portion of $F$.

Presented to the Society, November 25, 1944; received by the editors October 2, 1944.

${ }^{1}$ Numbers in brackets refer to the references cited at the end of the paper. 
We shall use as lemmas the following known results.

LEMMA 1. If $u(x, y)$ is continuous at $\left(x_{0}, y_{0}\right)$ and harmonic in a deleted neighborhood of $\left(x_{0}, y_{0}\right)$, then $u(x, y)$ is harmonic at $\left(x_{0}, y_{0}\right)$.

Proof. This follows from the fact that the function can be expanded in a two-way power series in a deleted neighborhood of $\left(x_{0}, y_{0}\right)$.

LEMMA 2. If $u(x, y)$ is harmonic in the finite domain $D$, then for each rectangle $C(R)$ such that $R$ lies in $D$ we have

$$
\int_{C(R)} \frac{d u}{d \nu} d s=0
$$

where $d / d \nu$ denotes differentiation in the direction of the outward normal.

Proof. The result follows directly from Green's theorem,

$$
\int_{C(R)} \frac{d u}{d \nu} d s=\iint_{R}\left(\frac{\partial^{2} u}{\partial x^{2}}+\frac{\partial^{2} u}{\partial y^{2}}\right) d x d y .
$$

LEMмA 3. If $u(x, y)$ and its partial derivatives of the first order are continuous in $D$ and if for every square $C(Q)$ having sides parallel to the coordinate axes and such that $Q$ lies in $D$ we have

$$
\int_{C(Q)} \frac{d u}{d \nu} d s=0
$$

then $u(x, y)$ is harmonic in $D$.

Proof. The mean-value function,

$$
u^{(r)}(x, y) \equiv \frac{1}{4 r^{2}} \int_{-r}^{r} \int_{-r}^{r} u(x+\xi, y+\eta) d \xi d \eta
$$

has continuous partial derivatives of the second order in the part $D^{(r)}$ of $D$ in which $u^{(r)}(x, y)$ is defined. We have

$$
\frac{\partial^{2} u^{(r)}(x, y)}{\partial x^{2}}=\frac{1}{4 r^{2}} \int_{-r}^{r}\left[\frac{\partial u(x+r, y+\eta)}{\partial x}-\frac{\partial u(x-r, y+\eta)}{\partial x}\right] d \eta
$$

and

$$
\frac{\partial^{2} u^{(r)}(x, y)}{\partial y^{2}}=\frac{1}{4 r^{2}} \int_{-r}^{r}\left[\frac{\partial u(x+\xi, y+r)}{\partial y}-\frac{\partial u(x+\xi, y-r)}{\partial y}\right] d \xi,
$$

whence 


$$
\Delta u^{(r)}(x, y)=\frac{1}{4 r^{2}} \int_{C\left(Q_{r}\right)} \frac{d u}{d \nu} d s,
$$

where $C\left(Q_{r}\right)$ is the square with sides of length $2 r$ and parallel to the coordinate axes, and with center at $(x, y)$.

From (3) and (4) it follows that $u^{(r)}(x, y)$ is harmonic in $D^{(r)}$. Hence $u(x, y)$, the uniform limit of $u^{(r)}(x, y)$ on any closed and bounded subset of $D$ as $r \rightarrow 0$, is harmonic in $D$.

Lemma 4 (BAIRE's TheOREM). Let $F$ be a non-null plane set lying in a domain $D$ and closed with respect to $D$, and let $\left\{F_{n}\right\}, n=1,2, \cdots$, be a sequence of sets lying in $D$ and closed with respect to $D$ such that $\left\{F_{n}\right\}$ covers $F$; that is, each point of $F$ is a point of at least one $F_{n}$. Then there is a member $F_{N}$ of $\left\{F_{n}\right\}$ which contains a portion of $F$.

Proof. If the result were not valid, there would be a descending sequence $\left\{P_{n}\right\}, P_{1} \supset P_{2} \supset \cdots$, of portions of $F$ such that $P_{n}$ and $F_{n}$ have no point in common, $n=1,2, \cdots$. Then $\prod_{n=1}^{\infty} P_{n}$ and $\sum_{n=1}^{\infty} F_{n}$ would have no point in common. But this is impossible since $\prod_{n=1}^{\infty} P_{n}$ contains a point of $F$ and $\sum_{n=1}^{\infty} F_{n}$ covers $F$.

LEMMA 5. Let $C(Q)$ be a square having sides parallel to the coordinate axes, let $F$ be a closed non-null set in $Q$, let $C(R)$ be the smallest rectangle (which may be degenerate) having sides parallel to the coordinate axes and satisfying the condition that $F$ is contained in $R$, and let the vertices of $C(R)$ have coordinates

$$
\left(x_{1}, y_{1}\right),\left(x_{2}, y_{1}\right),\left(x_{2}, y_{2}\right),\left(x_{1}, y_{2}\right), \quad x_{1} \leqq x_{2}, y_{1} \leqq y_{2} .
$$

If the real function $w(x, y)$ is defined on the set $Q$, if the first order partial derivatives of $w(x, y)$ exist, or even if the Dini derivates are finite, at every point of $Q$ except at most at the points of a denumerable set of points in $Q$, and if for the finite constant $N$ we have

$$
\begin{aligned}
& \left|w\left(x_{0}+h, y_{0}\right)-w\left(x_{0}, y_{0}\right)\right| \leqq N|h|, \\
& \left|w\left(x_{0}, y_{0}+k\right)-w\left(x_{0}, y_{0}\right)\right| \leqq N|k|,
\end{aligned}
$$

for all $\left(x_{0}, y_{0}\right)$ in $F$ and all $\left(x_{0}+h, y_{0}\right),\left(x_{0}, y_{0}+k\right)$ in $Q$, then

$$
\begin{aligned}
& \left|\int_{x_{1}}^{x_{2}}\left[w\left(x, y_{2}\right)-w\left(x, y_{1}\right)\right] d x-\iint_{F} \frac{\partial w}{\partial y} d x d y\right| \leqq 5 N \text { meas }(Q-F), \\
& \left|\int_{y_{1}}^{y_{2}}\left[w\left(x_{2}, y\right)-w\left(x_{1}, y\right)\right] d y-\iint_{F} \frac{\partial w}{\partial x} d x d y\right| \leqq 5 N \text { meas }(Q-F) .
\end{aligned}
$$

Proof. A proof of Lemma 5 may be found in Saks [5, pp. 198-199] or Menchoff [3, pp. 10-12]. 
We note, relative to Lemma 5 , that since $w(x, y)$ is differentiable, or has finite Dini derivates, with respect to $x$ and $y$ at all points of $Q$ except at most at the points of a denumerable set of points in $Q$, it follows $[5$, pp. 236, 272] that $\partial w / \partial x$ and $\partial w / \partial y$ exist almost everywhere in $Q$ and are integrable.

3. Theorem. We shall establish the following result.

THEOREM. If the real function $u(x, y)$ and its first order partial derivatives with respect to $x$ and $y$ are continuous in the finite domain $D$, and if $\partial u / \partial x$ and $\partial u / \partial y$ are differentiable, or even have finite Dini derivatives, with respect to $x$ and $y$ at all points of $D$ except at most at the points of a denumerable set of points in $D$, and if the Laplace equation,

$$
\Delta u \equiv \partial^{2} u / \partial x^{2}+\partial^{2} u / \partial y^{2}=0,
$$

is satisfied at almost all points at which $\partial^{2} u / \partial x^{2}$ and $\partial^{2} u / \partial y^{2}$ *ist in $D$, then $u(x, y)$ is harmonic in $D$.

Proof. Suppose that there is a point of $D$ at which $u(x, y)$ is not harmonic; we shall obtain a contradiction.

Denote the set of points of $D$ at which $u(x, y)$ is not harmonic by $F$. Since by definition the set of points at which $u(x, y)$ is harmonic is open, it follows that $F$ is closed with respect to $D$. Further, by Lemma $1, F$ has no isolated points. Hence $F$ is perfect with respect to $D$.

For each positive integer $n$, let $F_{n}$ be the set of points $(x, y)$ of $F$ for which

$$
\begin{aligned}
& \left|\frac{\partial u(x+h, y)}{\partial x}-\frac{\partial u(x, y)}{\partial x}\right| \leqq n|h|, \\
& \left|\frac{\partial u(x, y+h)}{\partial x}-\frac{\partial u(x, y)}{\partial x}\right| \leqq n|h|, \\
& \left|\frac{\partial u(x+h, y)}{\partial y}-\frac{\partial u(x, y)}{\partial y}\right| \leqq n|h|, \\
& \left|\frac{\partial u(x, y+h)}{\partial y}-\frac{\partial u(x, y)}{\partial y}\right| \leqq n|h|,
\end{aligned}
$$

for all $h$ satisfying $|h| \leqq 1 / n$. Since $\partial u / \partial x$ and $\partial u / \partial y$ are continuous in $D$, it follows that the sets $F_{n}$ are closed with respect to $D$; and since $\partial u / \partial x$ and $\partial u / \partial y$ are differentiable, or have finite Dini derivates, with respect to $x$ and $y$ at all points of $D$ except at most at the points of a denumerable set of points in $D$, it follows that the sets $\left\{F_{n}\right\}$, 
$n=1,2, \cdots$, cover all of $F$ except the points of a set $H$ which is at most denumerable.

Then we have

$$
F=\sum_{n=1}^{\infty} F_{n}+H
$$

It follows from (5) and Lemma 4 that thee is a portion $P$ of $F$ either consisting of a single isolated point of $H$, or contained in an $F_{N}$ of $\left\{F_{n}\right\}$. But since $F$ is perfect with respect to $D$, the former alternative is impossible, so that the latter alternative holds.

The above portion $P$ of $F$ is contained in $F_{N}$, and is the common part of $F$ and a set $Q_{0}$ in $D$, where $C\left(Q_{0}\right)$ is a square in $D$ with center at a point of $F$ and with sides parallel to the coordinate axes.

Let $C(Q)$ be any square lying in $Q_{0}$ and having its sides parallel to the coordinate axes, and let $F \cdot Q$ be the common part of $F$ and $Q$. Let the sides of $C(Q)$ be divided into $n$ equal parts, with $n$ so large that the length of each part is less than or equal to $1 / N$. Lines through the points of division parallel to the coordinate axes divide $Q$ into $n^{2}$ squares. Let $Q_{p, n}, p=1,2, \cdots, l ; l \leqq n^{2}$, denote those of the $n^{2}$ squares having points in common with $F$.

For each $Q_{p, n}$ let $C\left(R_{p, n}\right)$ be the smallest rectangle (which may be degenerate) having sides parallel to the coordinate axes and such that $R_{p, n}$ contains $F \cdot Q_{p, n}$. Let the vertices of $C\left(R_{p, n}\right)$ have coordinates

$$
\begin{array}{cl}
\left(x_{1, p, n}, y_{1, p, n}\right), & \left(x_{2, p, n}, y_{1, p, n}\right), \quad\left(x_{2, p, n}, y_{2, p, n}\right), \quad\left(x_{1, p, n}, y_{2, p, n}\right), \\
& x_{1, p, n} \leqq x_{2, p, n}, y_{1, p, n} \leqq y_{2, p, n} .
\end{array}
$$

By Lemma 2 and the uniform continuity of $u(x, y)$ in $Q$, we have

$$
\int_{C(Q)} \frac{d u}{d \nu} d s=\sum_{p=1}^{l} \int_{C\left(R_{p, n}\right)} \frac{d u}{d \nu} d s .
$$

Since

$$
\begin{aligned}
& \int_{C\left(R_{p, n}\right)} \frac{d u}{d \nu} d s=\int_{x_{1, p, n}}^{x_{2, p, n}} {\left[\frac{\partial u\left(x, y_{2, p, n}\right)}{\partial y}-\frac{\partial u\left(x, y_{1, p, n}\right)}{\partial y}\right] d x } \\
&+\int_{y_{1, p, n}}^{y_{2, p, n}}\left[\frac{\partial u\left(x_{2, p, n}, y\right)}{\partial x}-\frac{\partial u\left(x_{1, p, n}, y\right)}{\partial x}\right] d y,
\end{aligned}
$$

by Lemma 5 we have

$$
\begin{aligned}
\mid \int_{C\left(R_{p, n}\right)} \frac{d u}{d \nu} d s-\iint_{F \cdot Q_{p, n}}\left(\frac{\partial^{2} u}{\partial x^{2}}+\right. & \left.\frac{\partial^{2} u}{\partial y^{2}}\right) d x d y \mid \\
& \leqq 10 N \text { meas }\left(Q_{p, n}-F \cdot Q_{p, n}\right) .
\end{aligned}
$$


From (6) and (7) we obtain

(8)

$$
\begin{aligned}
\mid \int_{C(Q)} \frac{d u}{d \nu} d s-\iint_{F \cdot Q}\left(\frac{\partial^{2} u}{\partial x^{2}}\right. & \left.+\frac{\partial^{2} u}{\partial y^{2}}\right) d x d y \mid \\
& \leqq 10 N \text { meas }\left(\sum_{p=1}^{l} Q_{p, n}-F \cdot Q\right) .
\end{aligned}
$$

Since the lengths of the sides of the squares $C\left(Q_{p, n}\right) \rightarrow 0$ as $n \rightarrow \infty$, it follows that

$$
\lim _{n \rightarrow \infty} \operatorname{meas}\left(\sum_{p=1}^{l} Q_{p, n}-F \cdot Q\right)=0 .
$$

By hypothesis and the note at the end of $\$ 2,(1)$ is satisfied almost everywhere in $D$. Consequently from (8) and (9) we obtain

$$
\int_{C(Q)} \frac{d u}{d \nu} d s=0 .
$$

Hence by Lemma $3, u(x, y)$ is harmonic in $Q_{0}$. But the center of $Q_{0}$ is a point of $F$, so that $u(x, y)$ is not harmonic in $Q_{0}$. Thus the supposition that there is a point of $D$ at which $u(x, y)$ is not harmonic has led to a contradiction.

\section{REFERENCES}

1. E. Hopf, Bemerkungen zur Aufgabe 49, Jber. Deutschen Math. Verein. vol. 39 (1930) part 2, pp. 4-6.

2. P. T. Maker, Conditions on $u(x, y)$ and $v(x, y)$ necessary and sufficient for the regularity of $u+i v$, Trans. Amer. Math. Soc. vol. 45 (1939) pp. 265-275.

3. D. Menchoff, Les conditions de monogénéitê, Actualités Scientifiques et Industrielles, no. 329, Paris, 1936.

4. J. Ridder, Harmonische, subharmonische und analytische Funktionen, Annali Scuola Normale Superiore, Pisa, (2) vol. 9 (1940) pp. 277-287.

5. S. Saks, Theory of the integral, New York, 1937.

The University of Texas 